\title{
Angiogenesis and vasculogenic mimicry are inhibited by 8-Br-cAMP through activation of the cAMP/PKA pathway in colorectal cancer
}

This article was published in the following Dove Press journal:

OncoTargets and Therapy

\author{
Sen Wang* \\ Zhiyuan Zhang* \\ Wenwei Qian \\ Dongjian Ji \\ Qingyuan Wang \\ Bing Ji \\ Yue Zhang \\ Chuan Zhang \\ Ye Sun \\ Chunyan Zhu \\ Yueming Sun \\ Department of Colorectal Surgery, \\ The First Affiliated Hospital of \\ Nanjing Medical University, Nanjing, \\ Jiangsu 210029 , China \\ *These authors contributed equally \\ to this work
}

\begin{abstract}
Introduction: Vasculogenic mimicry (VM) describes the formation of an epithelial-independent tumor microcirculation system that differs from traditional angiogenesis. Angiogenesis and the formation of VM are closely related through the cyclic adenosine monophosphate (cAMP)/ protein kinase A (PKA) pathway and the epithelial-mesenchymal transition (EMT) process.

Materials and methods: In this study, 8-Br-cAMP, a cAMP analog and PKA activator, was used to activate the cAMP/PKA pathway to evaluate the effects of cAMP/PKA on angiogenesis and VM in colorectal cancer (CRC) cells. We used a syngeneic model of CRC in BALB/c mice.

Results: We discovered that treatment with 8-Br-cAMP significantly reduced tumor number compared to control mice after the 7th, 14th, and 28th days of treatment. VM was evaluated by periodic acid-schiff (PAS)-CD31 staining, and we found that VM was inhibited by 8-BrcAMP treatment in vivo. Immunohistochemistry confirmed the inhibition of vascular endothelial growth factor (VEGF) and cAMP and the activation of PKA by 8-Br-cAMP; quantitative real-time-PCR (qRT-PCR) demonstrated that 8-Br-cAMP regulated the expression of vascular endothelial (VE)-cadherin, matrix metalloproteinase 2 (MMP2), ephrin type-A receptor 2 (EphA2), and VEGF in vivo. Experiments in vitro revealed that treatment with 8-Br-cAMP and U0126 decreased VEGF expression through PKA-ERK in CT26 cells by qRT-PCR. We further confirmed that tube formation of human umbilical vein endothelial cells was inhibited by 8 -Br-cAMP in vitro.

Discussion: This study demonstrates that angiogenesis and VM are inhibited by 8-Br-cAMP treatment. Our data indicate that 8-Br-cAMP acts through the cAMP/PKA-ERK pathway and through EMT processes in CRC. These findings provide an insight into mechanisms of CRC and suggest that the cAMP/PKA-ERK pathway is a novel potential therapeutic target for the treatment of CRC
\end{abstract}

Keywords: vasculogenic mimicry, angiogenesis, VEGF, 8-Br-cAMP, cAMP

\section{Introduction}

Colorectal cancer (CRC) has the third highest incidence ${ }^{1}$ and the second highest mortality among all cancers worldwide. ${ }^{2}$ Liver metastasis is the leading cause of mortality in patients diagnosed with CRC. It is estimated that $\sim 50 \%$ of patients with CRC die from distant metastases.

Angiogenesis is a key event in the malignant progression of CRC and enables CRC tumor growth by providing the growing tumor with access to the blood supply. This process not only enhances cell proliferation but can also facilitate dissemination of CRC cells. Vascular endothelial growth factor (VEGF) is a central player in angiogenesis
Correspondence: Yueming Sun

Department of Colorectal Surgery,

The First Affiliated Hospital of Nanjing

Medical University, Guangzhou Road

No. 300, Nanjing, Jiangsu 210029,

People's Republic of China

$\mathrm{Tel}+86$ I $3505 \mid 888397$

Fax +86 02164085875

Email jssunyueming@I26.com 
and is one of the best characterized angiogenic factors. ${ }^{3}$ VEGF recruits and stimulates the proliferation of endothelial cells, directly leading to angiogenesis. ${ }^{4}$

It has been reported that there is a significant difference in VEGF expression between primary CRC tissue and metastatic liver lesions from CRC patients with liver metastases. ${ }^{5}$ We hypothesize that there are additional mechanisms underlying the establishment of tumor microcirculation in CRC beyond VEGF-driven angiogenesis.

Vasculogenic mimicry (VM), first described in melanoma by Maniotis et al, ${ }^{6}$ is the establishment of an epithelialindependent tumor microcirculation system that differs from traditional angiogenesis. In VM, vascular channels are composed of periodic acid-schiff (PAS)-positive basement membrane and CD31-negative epithelial-like colorectal cell. These vessels allow red blood cells to pass through, providing direct exposure of tumor cells to a blood supply and potentially increasing opportunities for metastatic dissemination. Since VM was first described in 1999, the occurrence of VM has been confirmed in ovarian cancer, breast cancer, glioblastoma, and liver cancer. ${ }^{7-9}$ We have reported that VM occurs in CRC and is associated with tumor node metastasis staging. Additionally, VM has been associated with metastasis and reduced survival in CRC patients. ${ }^{10}$

Cyclic adenosine monophosphate (cAMP) acts to convey extracellular signals inside cells, and the activity of protein kinase A (PKA) is dependent on cellular levels of cAMP. The cAMP/PKA pathway is a classical G-protein-coupled receptor-regulated pathway. In addition, the activation of the second messenger cAMP is correlated with VEGF expression. Amano et $\mathrm{al}^{11}$ found that in a rat sponge implanting model, the activation of cAMP promoted VEGF expression, resulting in neoplastic vascularization. A study by Namkoong et $\mathrm{al}^{12}$ revealed that adenylate cyclase-activating forskolin promoted human vascular epithelial cells to secret VEGF and form vasculature. Lissitzky et $\mathrm{al}^{13}$ reported that cAMP affects the formation of VM in cultured melanoma cells. Collectively, these studies indicate that angiogenesis and formation of VM are closely related through the cAMP/PKA pathway. Therefore, we hypothesized that the cAMP/PKA pathway may be a potential common pathway of angiogenesis and VM and that this axis may be a potential target to suppress the neovascularization of primary $\mathrm{CRC}$ and liver metastasis, thereby restricting CRC growth and improving survival outcomes for patients with CRC.

VM is regulated by vascular endothelial (VE)-cadherin, matrix metalloproteinases (MMPs), and ephrin type A receptor 2 (EphA2), which play roles in epithelial-mesenchymal transition $(\mathrm{EMT})^{14}$ and can contribute to extracellular matrix remodeling and the formation of $\mathrm{VM} .{ }^{15} \mathrm{EMT}$ is a dynamic process by which epithelial cancer cells are converted into dedifferentiated cells with additional mesenchymal properties $^{16}$ and can promote VM in cancer. VE-cadherin is an important adhesion protein that regulates vasculogenic activities. EphA2 is a member of the ephrin receptor family of PTKs and is of importance in angiogenesis. VE-cadherin regulates the expression and phosphorylation of EphA2 on the cell membrane. Phosphorylation of EphA2 in turn promotes the phosphorylation of VE-cadherin, resulting in the eventual loss of adhesion between cells and subsequently leading to cell migration and infiltration into VM. Hess et al ${ }^{17}$ reported that EphA2 promotes the progression of VM by dephosphorylating focal adhesion kinase and remodeling the extracellular matrix in melanoma. Wang et $\mathrm{al}^{3}$ reported that VEGF induces EphA2 to increase the expression of MMPs and remodel the extracellular matrix, leading to the regulation of VM. These findings also suggest that angiogenesis and VM may be correlated with EMT processes.

In the current study, we used 8-Br-cAMP, a cAMP analog and PKA activator, to activate the $\mathrm{cAMP} / \mathrm{PKA}$ pathway to evaluate the impact on angiogenesis and VM in CRC cells in vivo and in vitro and to explore the potential of targeting the $\mathrm{cAMP} / \mathrm{PKA}$ axis in the treatment of CRC.

\section{Material and methods}

\section{Cell line and syngeneic tumor formation}

Our cells were purchased from American Type Culture Collection (ATCC, Manassas, VA, USA). The human umbilical vein endothelial cells (HUVEC) originates from normal neonatal human umbilical vein endothelial cells, and the CT26 cell line was derived from colon carcinoma tissues of BALB/c mice according to ATCC websites. The CT26 CRC cell line, derived from a BALB/c murine adenocarcinoma, was maintained in Roswell Park Memorial Institute-1640 medium supplemented with $10 \%$ fetal bovine serum at $37^{\circ} \mathrm{C}$ and $5 \% \mathrm{CO}_{2}$. For passaging or the preparation of CT26 cells to inject into BALB/c mice, CT26 cells were dissociated with $0.25 \%$ trypsin and centrifuged for $5 \mathrm{~min}$ at 1,000 rpm. Following aspiration of the supernatant, phosphate buffer saline (PBS) was added and cell concentration was adjusted to $1 \times 10^{6}$ cells $/ \mathrm{mL}$.

Forty-two 4- to 5-week-old male BALB/c mice, weighing from 18 to $22 \mathrm{~g}$, were purchased from the Changzhou Kawensi Biotech Company (Changzhou, China). Six mice were injected subcutaneously in the back with $0.4 \mathrm{~mL}$ of the CT26 cell suspension ( $4 \times 10^{5}$ cells/mouse). Tumors measuring $1 \mathrm{~cm}$ in diameter emerged 10 days later in these mice. Pathological 
examination of tissue confirmed the presence of carcinoma. All tumor tissues were removed and sliced into pieces for serial implantation into the cecum of BALB/c mice.

Thirty-six mice received the implantation of CT26 carcinoma tissue in their cecum. After general anesthesia and sterilization, a $2 \mathrm{~cm}$ vertical incision was made at the right lower quadrant of the abdomen. The cecum was then pulled out of the abdomen. The serosa of the cecum that was exposed out was scratched, and a $2 \mathrm{~mm}$ diameter tumor tissue was attached with fibrin glue. Finally, the cecum was put back into place and the skin was sealed. After tumor implantation, mice were randomly divided into a control group and an experimental group. In the experimental group, the intraperitoneal injection of 8-Br-cAMP ( $60 \mathrm{mg} / \mathrm{kg} /$ day $)$ was performed for 7 days, while control mice received injection of normal saline. Mice were sacrificed on the 7th, 14th, and 28th days, and tumor tissue was harvested for the evaluation of gene expression. However, due to a high mortality rate, the number of mice for sacrifice at each time point was adjusted in order to guarantee that mice were available for culling on the 28th day. This study was approved by the Animal Ethics Committee of Northern Jiangsu People's Hospital, Yangzhou Clinical School of Nanjing Medical University. This study was also conducted following the guidelines and regulations of Animal Ethics Committee of Northern Jiangsu People's Hospital, Yangzhou Clinical School of Nanjing Medical University.

\section{Hematoxylin and eosin staining}

Paraffin-embedded sections of murine tumor tissues were prepared and stained with hematoxylin and eosin. Slices were cut into $5 \mu \mathrm{m}$ sections and stained with hematoxylin-eosin for histological evaluation under a microscope (Olympus Corporation, Tokyo, Japan) by two independent pathologists.

\section{Observation of VM}

Immunohistochemical staining with CD31/CD34 and PAS is used to identify VM in tumor tissues. ${ }^{18-20} \mathrm{VM}$ is recognized as vascular-like patterns of tumor cells gathered together without necrosis, hemorrhage, or infiltration of inflammatory cells. CD31/CD34 and PAS staining demonstrated that VM is composed of extracellular basement membrane (PAS positive) with red blood cells inside. Tumor cells were CD31 negative, while typical vessels showed CD31 positivity and PAS positivity. Therefore, the double staining could validate VM as it is PAS positive and CD31 negative. Five VM-rich areas were evaluated under $100 \times$ magnification in each section, and the quantity of VM in each area was calculated under $400 \times$ magnification. The mean value of all five areas is defined as VM density (VMD).

\section{Quantitative real-time-PCR ( $q R T-P C R)$}

Total RNA was isolated from cells using the TRIzol reagent (Thermo Fisher Scientific, Waltham, MA, USA). cDNAs were generated using the PrimeScript RT Master Mix kit (Takara, Dalian, China). Primers for qRT-PCR were designed using online tools available at the Primer3 website. qRT-PCR was performed on a StepOnePlus Real-Time PCR system (Thermo Fisher Scientific) using the SYBR Green PCR kit (Hoffman-La Roche Ltd., Basel, Switzerland) in a final volume of $10 \mu \mathrm{L}$. Relative quantification was performed for genes listed in Table 1. Each reaction was performed and repeated at least three times. The data were analyzed using the $2^{-\Delta \Delta \mathrm{Ct}}$ method.

\section{Immunohistochemistry analysis}

After animal sacrifice, tumor tissues obtained from CT26 tumor-bearing subjects were prepared in formalin-fixed, paraffin-embedded sections. Samples were cut into $4-\mu \mathrm{m}-$ thick serial sections, deparaffinized with xylene, and rehydrated in alcohol. Sections were subsequently submerged in antigen retrieval buffer and microwaved for antigen fixation. Sections were treated with hydrogen peroxide to block endogenous nonspecific binding activity and were incubated overnight at $4^{\circ} \mathrm{C}$ with diluted primary antibodies. Slides were incubated with the appropriate Horseradish Peroxidasepolymer-conjugated secondary antibodies at $37^{\circ} \mathrm{C}$ for $1 \mathrm{~h}$. The primary antibodies that were used are as follows: mouse anti-cAMP antibody (sc73761; Santa Cruz Biotechnology Inc., Dallas, TX, USA), mouse anti-PKA antibody (sc28315; Santa Cruz Biotechnology Inc.), rabbit anti-CD31 antibody (sc-1506; Santa Cruz Biotechnology Inc.), and rabbit antiVEGF antibody (sc-507; Santa Cruz Biotechnology Inc.).

Table I Primer sequence used for qRT-PCR

\begin{tabular}{ll}
\hline Primer & Sequence (5'-3') \\
\hline VE-cadherin & F 5'-GGCAATCAACTGTGCTCTCC-3' \\
& R 5'-GGCAATCAACTGTGCTCTCC-3' \\
EphA2 & F 5'-TTAGGGAGAAGGATGGTGAGTT-3' \\
& R 5'-GGTCGAGGGCATGGTGTA-3' \\
MMP2 & F 5'-CAGTGATGGCTTCCTCTGGT-3' \\
& R 5'-CAGTGATGGCTTCCTCTGGT-3' \\
$\beta$-Actin & F 5'-ATGTGGATCAGCAAGCAGGA-3' \\
PKA & R 5'-ATGTGGATCAGCAAGCAGGA-3' \\
& F 5'-CAACACAAGCCGACAAAAAGAC-3' \\
& R 5'-GATAGCGTAGGGAAACCAAGAA-3'
\end{tabular}

Abbreviations: EphA2, ephrin type-A receptor 2; F, forward; MMP2, matrix metalloproteinase 2; PKA, protein kinase A; qRT-PCR, quantitative RT-PCR; R, reverse; $\mathrm{VE}$, vascular endothelial. 
Negative control slides followed the same protocol, with the exception that primary antibodies were replaced with PBS. Subsequently, we stained sections with 3,3-diaminobenzidine solution for $3 \mathrm{~min}$ and counterstained the nuclei with hematoxylin. Stained tissue slices were reviewed and independently scored by two pathologists.

\section{Drug treatments}

Because we hypothesized that the cAMP/PKA-ERK angiogenesis axis plays an important role in the regulation of VM, the ERK inhibitor U0126 was used to inhibit the cAMP/PKAERK axis. CT26 and HUVEC cell cultures were divided into three groups: treatment with $10 \mu \mathrm{M} 8$-Br-cAMP, treatment with $10 \mu \mathrm{M}$ U0126 treatment, and untreated control. Treatment duration lasted $1 \mathrm{~h}$, after which total RNA was extracted.

\section{Tube formation assay}

We used HUVEC cells to process the tube formation assay. Matrigel (BD, Franklin Lakes, NJ, USA) and pipette tips were stored $4^{\circ} \mathrm{C}$ overnight prior to preparation of the tube formation assays. Fifty microliters of matrigel was added to prechilled 96-well plates on ice. When cells reached 70\%-80\% confluence, they were digested and then resuspended in serum-free dulbecco's modified eagle medium (DMEM) to a concentration of $2 \times 10^{5}$ cells $/ \mathrm{mL}$. A total of $50 \mu \mathrm{L}$ of cell suspension was added onto the matrigel of each well. 8-BrcAMP was added into serum-free DMEM. After overnight incubation at $37^{\circ} \mathrm{C}$, tube network formation was observed under the microscope and photographed, and the area and connectivity of the networks were calculated.

\section{Western blot}

Protein was extracted from CRC cells and tissues by using a RIPA kit (Beyotime, Shanghai, China) according to the manufacturer's protocol. Different molecular weights of protein were separated on $10 \%$ sodium dodecyl sulfate polyacrylamide gel electrophoresis gels in the running buffer and transferred to polyvinylidene fluoride membranes (EMD Millipore, Bedford, MA, USA) in the transfer buffer. Then, the membranes were blocked in 5\% nonfat milk for $>2 \mathrm{~h}$ and incubated in specific primary antibodies at $4{ }^{\circ} \mathrm{C}$ overnight. After washing in tris-buffered saline and Tweens for three times (10 min each time), the membranes were transferred to secondary antibodies (rabbit or mouse) at room temperature for $2 \mathrm{~h}$. Protein bands could be exposed by using ECL Plus (EMD Millipore) with a Bio-Imaging System. The specific primary antibodies were as follows: E-cadherin, VE-cadherin, EphA2, MMP2, PKA, VEGF, and Tubulin, which were used as an internal control.

\section{Results \\ 8-Br-cAMP significantly inhibits the growth of CT26 tumors in mice}

Initially, subcutaneous CT26 tumors were established in six male BALB/c mice (Figure 1A). Afterward, 36 mice received serial transplantation of tumor fragments into their cecum and were randomized to treatment with 8-Br-cAMP or control. Subjects in both groups were sacrificed for the observation of tumor progression on the 7 th, 14 th, and 28 th days (Figure 1B and C). Primary tumor nodules and abdominal metastatic lesions were counted and compared between both groups. Seven days after implantation, the mean amounts of primary CRC tumor nodules and liver metastases in the control group were 49.33 \pm 26.86 and 3.00 \pm 5.20 , respectively, while in the 8-Br-cAMP treatment group, the mean amounts of primary CRC tumor nodules and liver metastases were 3.75 \pm 2.63 and 0 , respectively. On the 14 th day, the mean amounts of primary tumor nodules in the control and 8-Br-cAMP groups were $53.25 \pm 12.58$ and $9.00 \pm 7.81$, respectively; the mean amounts of liver metastases in the control and 8-Br-cAMP groups were 6.75 \pm 5.91 and 0, respectively. Ascites with pyaemia and macrosopic liver metastases were observed in mice from control group on the 14th day. Subjects from the 8-Br-cAMP treatment group did not show signs of liver metastases, metastasis to other organs, or ascites on the 7 th and 14 th days. On the 28th day, an increased mean number of primary tumors and metastatic lesions were observed in the control group ( $78 \pm 29.03$ and $11.25 \pm 14.41$, respectively). In these mice, massive liver metastases were observed, and tumors were diffusively distributed throughout the abdomen. The abdominal cavity was enlarged and had massive pyemic ascites. In contrast, three mice from the 8-Br-cAMP-treated group had no metastatic lesions and a mean count of colorectal and intestinal tumors of 17.33 \pm 8.50 (Figure 1D). Counts of colorectal and intestinal tumors in mice from the 8-Br-cAMP group were significantly lower than the control group on the 7 th, 14 th, and 28 th days $(P<0.05)$. However, no significant difference was observed in liver metastasis between the two groups. These results demonstrate that 8-Br-cAMP has significant inhibitory effects on primary CRC growth.

\section{Treatment with 8-Br-cAMP inhibits VM}

VM was evaluated by PAS-CD31 staining of colorectal and intestinal tumors from control and experimental groups. VM is recognized as PAS positive (membrane matrix stained pink) and CD31 negative (membrane matrix lacking brown stain). Less VM was observed in the 8-Br-cAMP-treated group than in the control group (Figure 2A). VMD, calculated as the mean VM of five areas in a sample, was significantly 
A
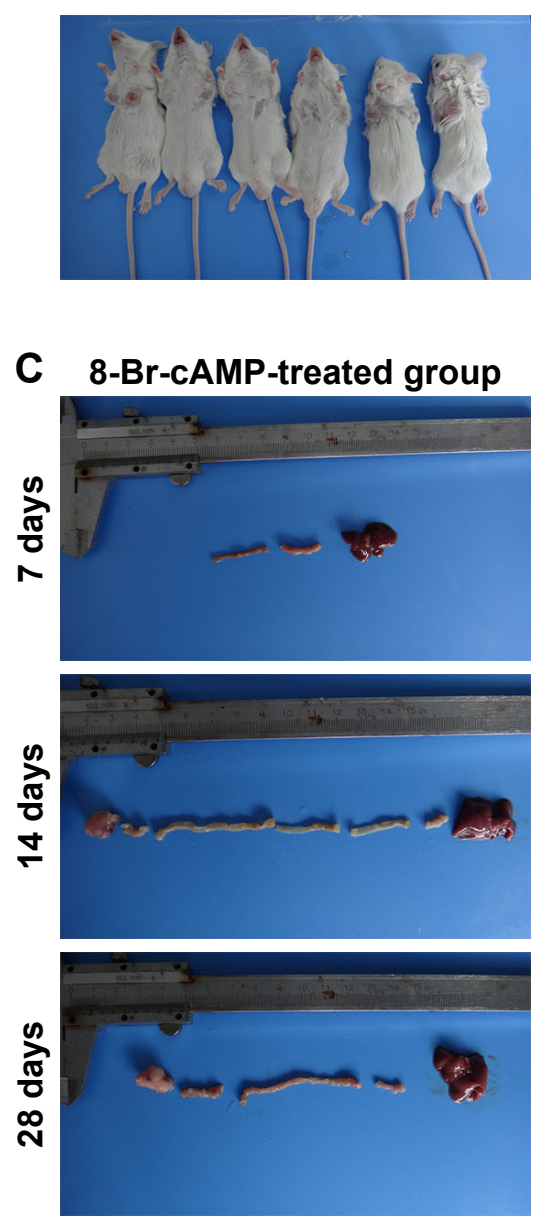

B

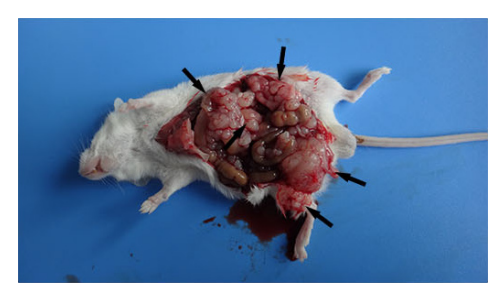

Control group
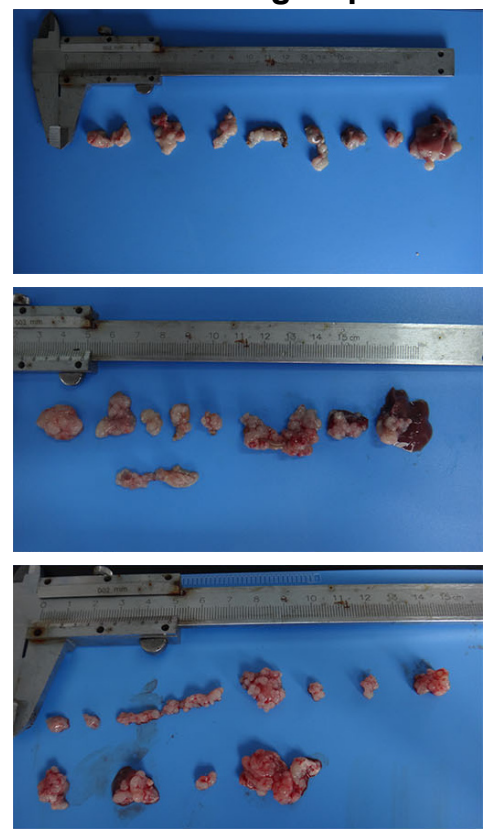

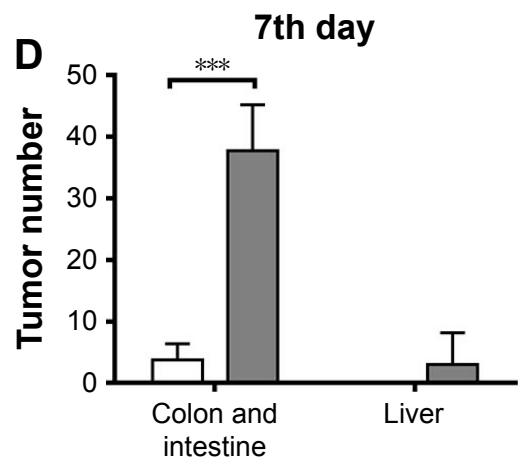

14th day

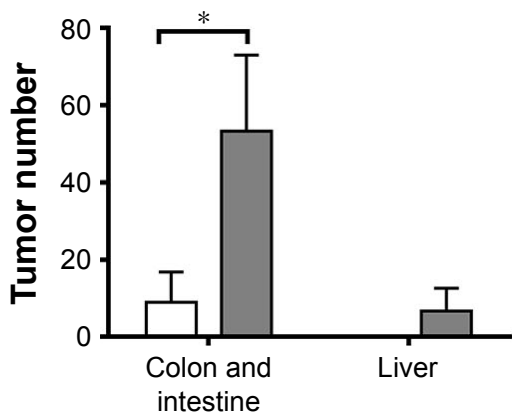

28th day

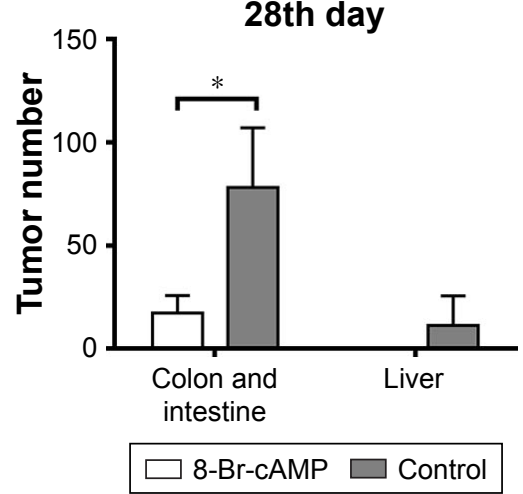

Figure I Syngeneic tumor formation in mice treated with 8-Br-cAMP vs control.

Notes: (A) Tumor formation was induced in six BALB/c mice after subcutaneous injection of CT26 cells ( $0.4 \mathrm{~mL} / \mathrm{per} \mathrm{mouse} ; 4 \times 10^{5}$ cells). (B) Representative image of a mouse demonstrating the serial implantation of CT26 tumors. (C) Primary and metastatic tumor formation of mice from 8-Br-cAMP-treated group and control group sacrificed on the 7th, 14th, and 28th days. (D) Tumor numbers were counted in mice sacrificed on the 7th, 14th, and 28th days. Counts of colorectal and intestinal tumors in mice from the 8 -Br-cAMP group were significantly lower than the control group. There were no significant differences in liver metastases. Significant differences: $* P<0.05$ and $* * * P<0.001$.

Abbreviation: cAMP, cyclic adenosine monophosphate.

lower in the 8-Br-cAMP group than in the control group throughout the experiment $(P<0.05$; Figure $2 \mathrm{~B})$. These results demonstrate that, in addition to inhibiting tumor growth, disruption of the cAMP/PKA axis with 8-Br-cAMP regulates the formation of $\mathrm{VM}$.

\section{Effects of 8-Br-cAMP treatment on VEGF, cAMP, and PKA}

$\mathrm{H} \& \mathrm{E}$ staining demonstrated successful in situ tumor formation in the murine model (Figure 3A); the presence of carcinoma was confirmed independently by two pathologists. The expression of VEGF, cAMP, and PKA in tumor tissues from control and 8-Br-cAMP-treated groups was evaluated through immunohistochemistry (IHC), as previously described (Figure 3B). The expression of cAMP was significantly lower in 8-Br-cAMP-treated group than in the control group, indicating that the cAMP analog, 8-Br-cAMP, inhibits cAMP expression. The expression of PKA in the 8-Br-cAMP group was significantly higher than that in the control group, confirming that PKA was activated by treatment with 8-Br-cAMP. The expression of VEGF in CRC tissues was significantly lower in the 8-Br-cAMP-treated group than in the control group, demonstrating that this key factor of angiogenesis was inhibited by the PKA activator, 

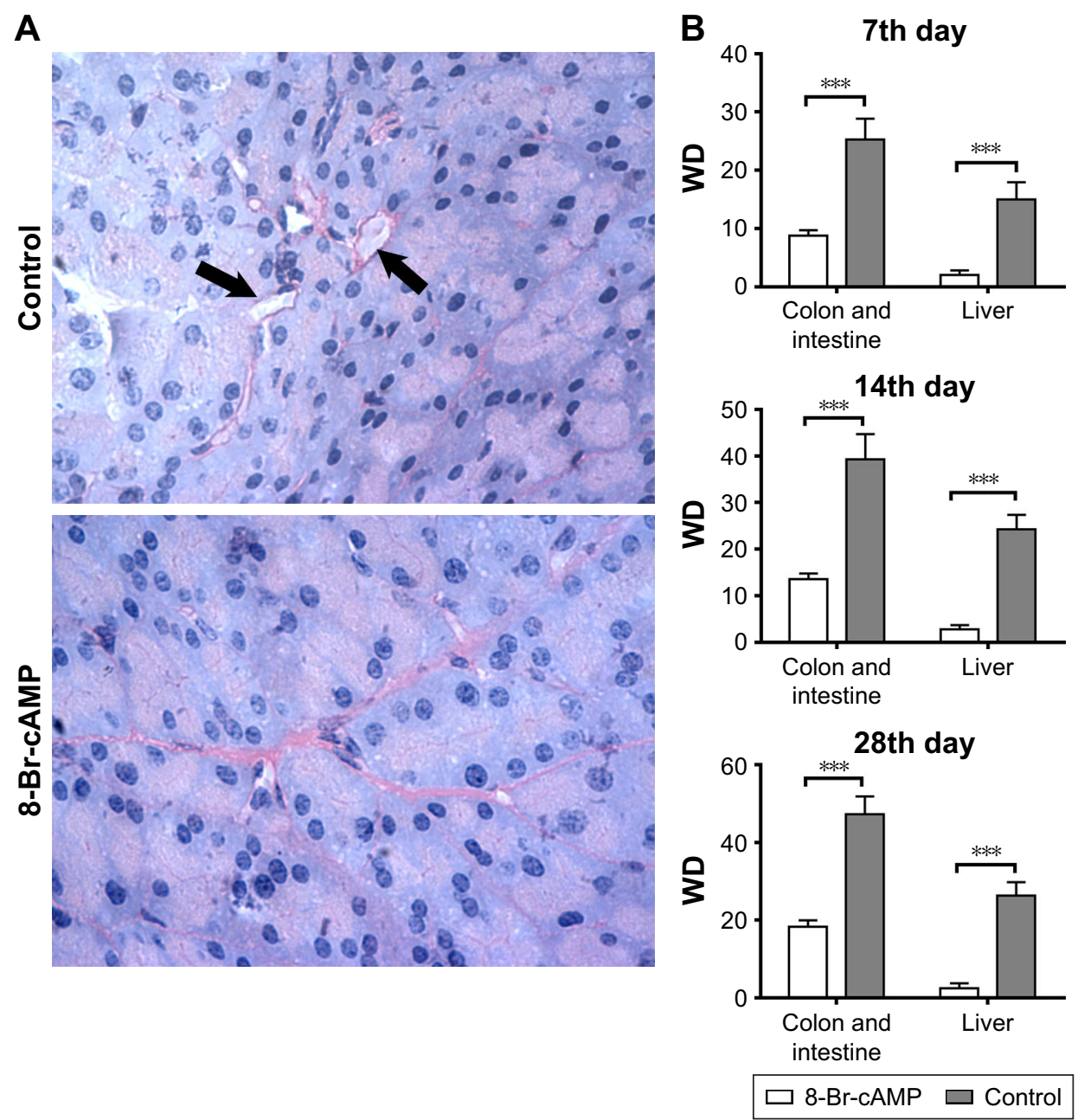

Figure 2 Formation of VM between 8-Br-cAMP-treated group and control group.

Notes: (A) PAS-CD3I staining of sections of colorectal and intestinal tumors from control and experimental groups. VM is recognized as PAS positive (membrane matrix stained pink) and CD3I negative (membrane matrix lacking brown stain). Black arrows indicate PAS-positive/CD3I-negative stained VM channels. In each section, the mean value of VM of five areas was calculated. Original magnification: $\times 400$. (B) VMD was also calculated in tissues from mice sacrificed on the 7 th, I4th, and 28 th days. Significant differences: $* * * P<0.001$.

Abbreviations: cAMP, cyclic adenosine monophosphate; PAS, periodic acid-schiff; VM, vasculogenic mimicry; VMD, VM density.

8-Br-cAMP. Lower expression of VEGF could lead to a decreased angiogenesis, diminishing neovasculature that promotes tumor growth. VEGF levels were positively correlated with VM and reduced following treatment with 8-Br-cAMP, indicating that VEGF and VM are regulated by 8-Br-cAMP and the cAMP/PKA axis.

\section{Regulation of EMT and VEGF expressions by 8 -Br-cAMP in vivo evaluated by qRT-PCR and Western blot}

qRT-PCR and Western blot were performed on CRC tissues obtained from $\mathrm{BALB} / \mathrm{c}$ mice from the 8-Br-cAMP treatment and control groups after sacrifice to determine the expressions of EMT-associated genes, including VE-cadherin, EphA2, and MMP2. qRT-PCR, and Western blot were performed on tissues obtained on the 14th and 28th days (Figure 3C). On the 14th day, there was no significant difference in expression detected in these three genes between the 8-BrcAMP-treated group and the control group (Figure 3C). However, on the 28th day, the expression of VE-cadherin and EphA2 was significantly lower in the 8-Br-cAMP-treated group than in the control group $(P<0.05$; Figure $3 \mathrm{C})$. This indicates that 8-Br-cAMP may have long-term effects on EMT processes, and the inhibitory influence may be exerted gradually. However, the MMP2 levels were not significantly different between the 8-Br-cAMP-treated group and the control group. Nevertheless, there was a trend that the expression of these genes was diminished over time in the 8-BrcAMP-treated group by the 28th day, and lack of statistical significance at the 14th day may be due to the short follow-up 
A

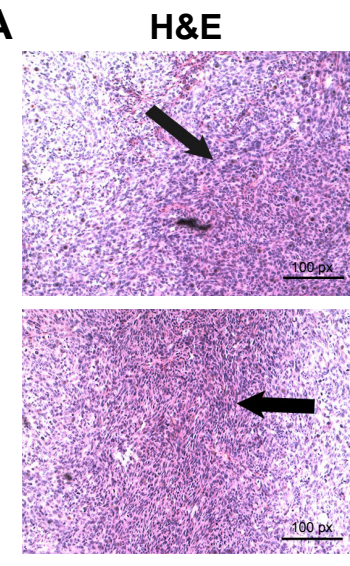

B

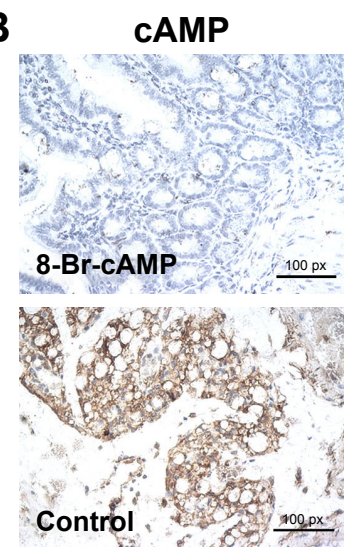

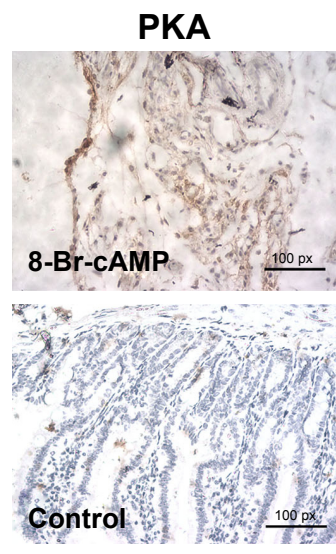

VEGF

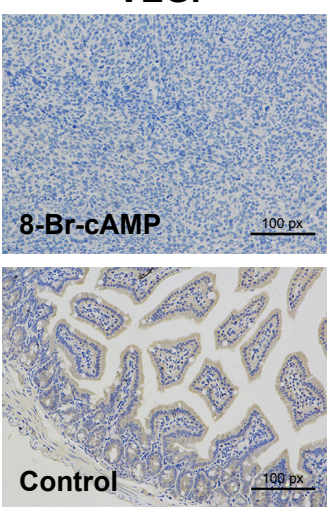

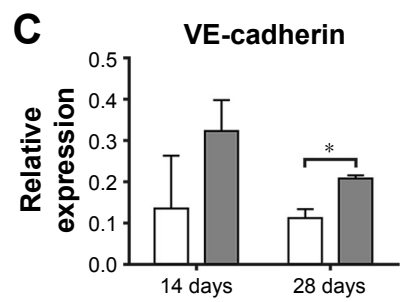
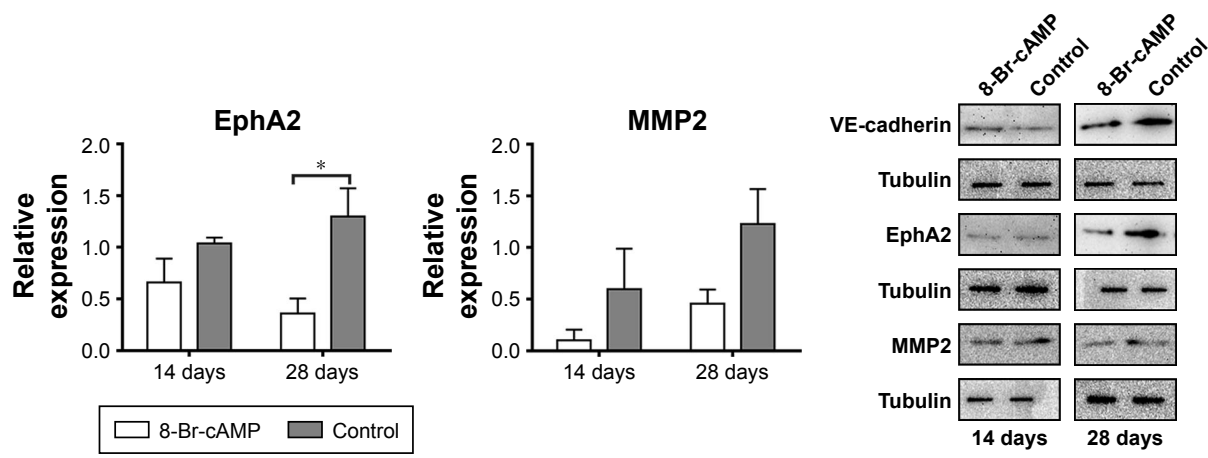

Figure 3 Functions of 8-Br-cAMP treatment on VEGF, cAMP, and PKA.

Notes: (A) H\&E staining of tumor tissues confirmed carcinoma formation in subjects from both treatment and control groups. Black arrows indicate atypia of cells. (B) IHC analysis revealed the expression of cAMP, PKA, and VEGF. Original magnification: $\times 200$. (C) The expression of VE-cadherin, EphA2, and MMP2 was detected by qRT-PCR and Western blot. Significant difference: $* P<0.05$.

Abbreviations: cAMP, cyclic adenosine monophosphate; EphA2, ephrin type A receptor 2; MMP2, matrix metalloproteinase 2; PKA, protein kinase A; qRT-PCR, quantitative RT-PCR; VE, vascular endothelial; VEGF, VE growth factor.

time and small sample size. Moreover, the significant differences we observed may be more pronounced if we continued the observation for a longer follow-up time. These data demonstrate that 8-Br-cAMP regulates EMT processes, inhibiting the expression of VE-cadherin and EphA2; this regulation correlates with the inhibition of angiogenesis and VM.

\section{qRT-PCR and Western blot evaluation of VEGF expression after treatment with 8-Br-cAMP and U0I26 in vitro}

To further elucidate the pathways and mechanisms associated with VM in CRC cells, we conducted in vitro experiments on the CT26 cell line. CT26 cells were divided into the following three groups: 8-Br-cAMP treatment, U0126 treatment, and negative control. qRT-PCR and Western blot demonstrated that 8-Br-cAMP significantly enhanced PKA expression, corresponding to the results of the IHC analysis on in vivo tumors treated with 8-Br-cAMP $(P<0.001$; Figure 4A). Importantly, the expressions of VEGF, VEcadherin, MMP2, and EphA2 were significantly lower in CT26 cells treated with 8-Br-cAMP than in the control group $(P<0.05$; Figure 4A), demonstrating that PKA can regulate EMT processes. Moreover, when CT26 cells were treated with U0126, the expression of VEGF, VE-cadherin, MMP2, and EphA2 was decreased relative to the control group. These findings demonstrated that 8-Br-cAMP is an analog of cAMP can activate PKA and further affect ERK, U0126 can function as a ERK inhibitor; thus, angiogenesis and EMT in CRC cells can be mediated through the PKAERK axis $(P<0.05)$.

\section{Inhibition of tube formation by $8-B r-c A M P$}

As Figure 4B described, HUVEC cells treated with 8-BrcAMP form fewer polygonal networks from individual cell bodies than the control group, directly confirming that angiogenesis is inhibited by the 8-Br-cAMP. Also, cells treated with U0126 generate fewer polygonal networks compared to the control. This is consistent with the reduction in VEGF expression following 8-Br-cAMP and U0126 treatment, as VEGF is a critical mediator of angiogenesis. These data suggest that 8-Br-cAMP and U0126 significantly abolish angiogenesis through the cAMP/PKA-ERK pathway. 
A
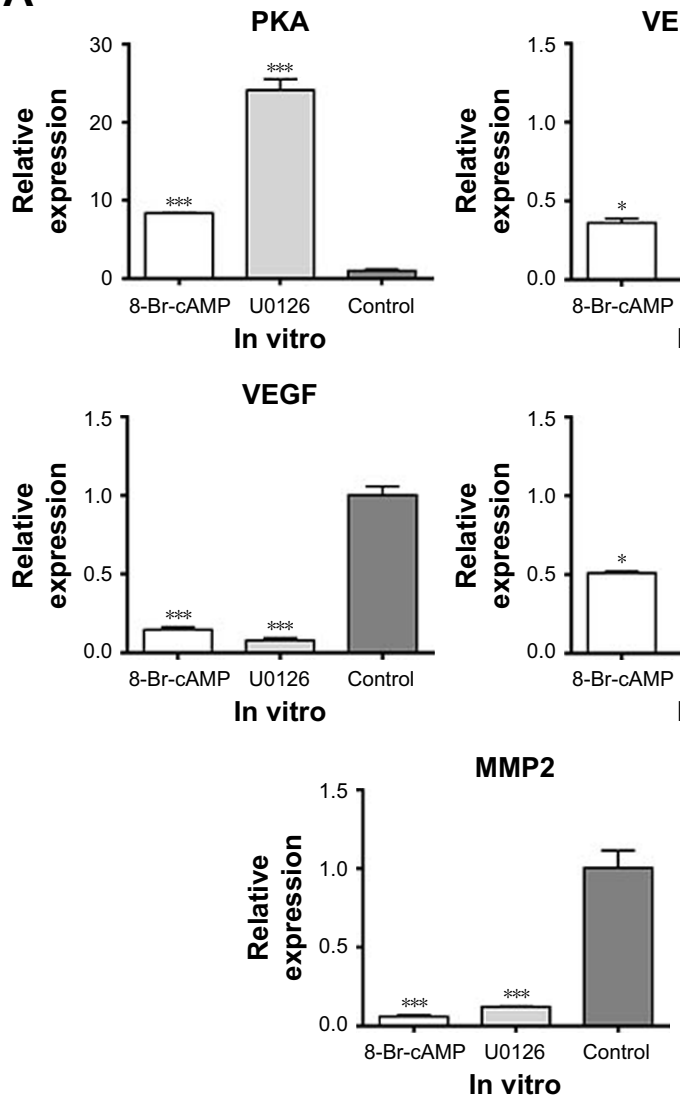

VE-cadherin

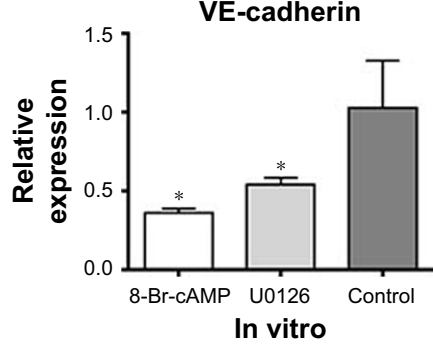

EphA2

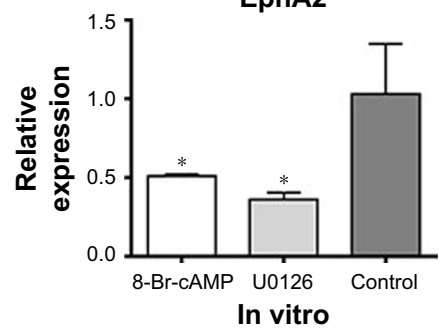

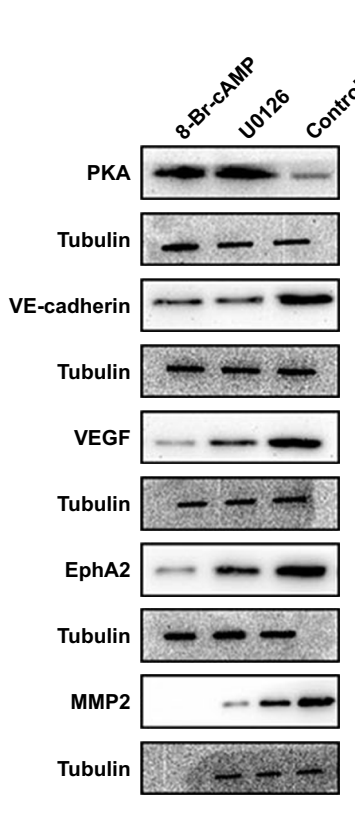

B
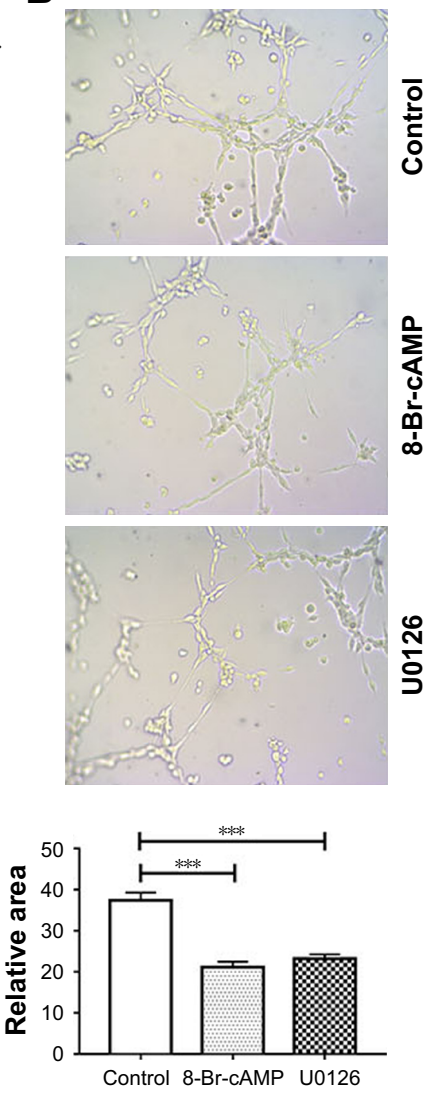

Figure 4 Expression of VEGF after treatment with 8-Br-cAMP and U0I26 in vitro.

Notes: (A) The expression of PKA, VE-cadherin, VEGF, EphA2, and MMP2 in vitro was detected by quantitative RT-PCR and Western blot. Significant differences: $* P<0.05$ and $* * * P<0.00 \mathrm{I}$. (B) Tube formation assay was performed to detect angiogenesis; original magnification: $\times 100$. CT26 cells treated with 8 -Br-cAMP form fewer polygonal networks than control cells. Signifcant differences: $* * * P<0.001$.

Abbreviations: cAMP, cyclic adenosine monophosphate; EphA2, ephrin type A receptor 2; MMP2, matrix metalloproteinase 2; PKA, protein kinase A; VE, vascular endothelial; VEGF, VE growth factor.

\section{Discussion}

$\mathrm{VM}$ is associated with poor prognosis in several malignant tumors, including glioblastoma, melanoma, and breast cancer ${ }^{18}$ and VEGF is reported to be closely related to the formation of VM. We conducted a series of in vivo and in vitro experiments to determine the impact of treatment with 8-Br-cAMP, a cAMP analog, on angiogenesis and VM in CRC. In vivo, 8-Br-cAMP exerted potent antitumor effects in CRC tumor-bearing BALB/c mice, indicating that activation of the cAMP/PKA pathway has an impact on tumor growth. Levels of VM and VEGF expression were significantly lower in 8-Br-cAMP-treated mice than in nontreated mice. The reductions corresponded to observations of tumor number and size. This suggested that reduced blood supply is directly related to the reduced tumor burden resulting from treatment with 8-Br-cAMP. Other report indicates the antiproliferative effects of treating cells with 8-BrcAMP ${ }^{21}$ indicating its strong antitumor potential.

While Lissitzky et $\mathrm{al}^{13}$ mentioned that PKA is not involved in the VM process, our results demonstrate that the
PKA activator, 8-Br-cAMP, inhibits the formation of VM, indicating that there may be a PKA-dependent regulation of VM and angiogenesis. It has also been reported that forskolin, a potent and specific activator of adenylyl cyclase, rather than PKA, significantly increases VEGF expression, ${ }^{12}$ proliferation, and migration; this is in contrast to our results. However, it was also reported that forskolin inhibits cell growth, making the role of forskolin on VM and angiogenesis controversial. ${ }^{22}$ The diverse actions of these drugs may be attributed to their different activation targets, and Namkoong et al ${ }^{12}$ reported that forskolin not only increases intracellular cAMP but also participates in the PKA pathway. Forskolin increases levels of cAMP, leading to the activation of PKA and subsequent activation of associated downstream targets through PKA. Forskolin may also activate downstream targets through elevated cAMP levels, rather than PKA; this may partially contribute to the diverse outcomes observed with forskolin treatment. In terms of 8-Br-cAMP, forskolin can only activate PKA by competing with 8 -Br-cAMP. However, the 
underlying mechanisms by which these two drugs lead to diverse outcomes are still unknown, and the diversity of the PKA and cAMP pathways is worth further study.

Hyper-activation of the ERK pathway, which is the downstream of the cAMP/PKA pathway, is a major cause of human cancers. ${ }^{23}$ In our research, 8-Br-cAMP reduced the expression of ERK through the cAMP/PKA pathway, which is concordant with previous report. ${ }^{24} \mathrm{It}$ is also reported that inhibition of ERK reduces the formation of VM and the expression of VEGF; our results are consistent with these reports. ${ }^{13}$ However, the activation of downstream ERK may be caused not only by the activation of PKA but also by elevated intracellular cAMP levels, ${ }^{3}$ as ERK is regulated by both pathways. While it is clear that ERK participates in the formation of VM and in the regulation of VEGF expression, ${ }^{20}$ the specific upstream factors contributing to ERK activation require further elucidation.

A regulatory relationship between angiogenesis and VM has been described. ${ }^{19,25}$ During tumorigenesis, VEGF is released by proteases, such as MMP2, and induces angiogenesis. ${ }^{26}$ Enhanced VEGF expression promotes the formation of VM, and EphA2 expression was also increased along with increased production of VEGF. ${ }^{25}$ It has also been reported that EMT-associated genes are highly involved in VM, including VE-cadherin, EphA2, and MMPs. ${ }^{15,17,27-30}$ According to previous report and our own research, the expression of these EMT-associated genes is significantly decreased along with changes in VEGF and VM when cells are treated with 8-Br-cAMP, indicating that EMT is positively correlated with VM and VEGF expression. ${ }^{31}$ Our data also revealed an association in the expression of ERK1/2 and VE-cadherin, a marker of VM formation, indicating that EMT is associated with the activity of the PKA-ERK axis, and this is consistent with previous reports. ${ }^{14,15}$

We selected the CT26-BALB/c syngeneic model of CRC as our in vivo model to simulate natural tumor growth in the presence of an intact immune system. However, serial implantation may have facilitated rapid tumor formation and growth, even in the presence of an immune system, resulting in more rapid mortality in subjects than we anticipated. In addition, the relatively small sample size in our study may have introduced bias into our results and was a limitation of our study.

Collectively, this study demonstrates that 8-Br-cAMP regulates angiogenesis and VM through the cAMP/PKA pathway and through downstream activation of the ERK signaling pathway in CRC in vitro and in vivo. We also confirmed that the expression of EMT markers (VE-cadherin and EphA2) and VEGF is associated with the formation of VM. To the best of our knowledge, this is the first study to evaluate the effect of activating the cAMP/PKA pathway with 8-Br-cAMP on the formation of VM and angiogenesis in CRC. This research indicates the potential importance of the cAMP/PKA pathway as a novel therapeutic target for the treatment of CRC.

\section{Acknowledgment}

This study was partially supported by the Jiangsu Key Medical Discipline (General Surgery) (ZDXKA2016005).

\section{Disclosure}

The authors report no conflicts of interest in this work.

\section{References}

1. Tan IB, Malik S, Ramnarayanan K, et al. High-depth sequencing of over 750 genes supports linear progression of primary tumors and metastases in most patients with liver-limited metastatic colorectal cancer. Genome Biol. 2015;16:32.

2. Hur K, Toiyama Y, Okugawa Y, et al. Circulating microRNA-203 predicts prognosis and metastasis in human colorectal cancer. Gut. 2017; 66(4):654-665.

3. Wang JY, Sun T, Zhao XL, et al. Functional significance of VEGF-a in human ovarian carcinoma: role in vasculogenic mimicry. Cancer Biol Ther. 2008;7(5):758-766.

4. Yang J, Shi QD, Song TB, et al. Vasoactive intestinal peptide increases VEGF expression to promote proliferation of brain vascular endothelial cells via the cAMP/PKA pathway after ischemic insult in vitro. Peptides. 2013;42:105-111.

5. Chen J, Li Q, Wang C, Wu J, Zhao G. Prognostic significance of c-erbB-2 and vascular endothelial growth factor in colorectal liver metastases. Ann Surg Oncol. 2010;17(6):1555-1563.

6. Maniotis AJ, Folberg R, Hess A, et al. Vascular channel formation by human melanoma cells in vivo and in vitro: vasculogenic mimicry. Am J Pathol. 1999;155(3):739-752.

7. Hendrix MJ, Seftor EA, Kirschmann DA, Seftor RE. Molecular biology of breast cancer metastasis. Molecular expression of vascular markers by aggressive breast cancer cells. Breast Cancer Res. 2000;2(6): 417-422.

8. Sood AK, Seftor EA, Fletcher MS, et al. Molecular determinants of ovarian cancer plasticity. Am J Pathol. 2001;158(4):1279-1288.

9. Liu XM, Zhang QP, Mu YG, et al. Clinical significance of vasculogenic mimicry in human gliomas. J Neurooncol. 2011;105(2):173-179.

10. Li W, Zong S, Shi Q, Li H, Xu J, Hou F. Hypoxia-induced vasculogenic mimicry formation in human colorectal cancer cells: involvement of HIF-1a, Claudin-4, and E-cadherin and Vimentin. Sci Rep. 2016;6: 37534.

11. Amano H, Ando K, Minamida S, et al. Adenylate cyclase/protein kinase A signaling pathway enhances angiogenesis through induction of vascular endothelial growth factor in vivo. Jpn J Pharmacol. 2001;87(3):181-188.

12. Namkoong S, Kim CK, Cho YL, et al. Forskolin increases angiogenesis through the coordinated cross-talk of PKA-dependent VEGF expression and Epac-mediated PI3K/Akt/eNOS signaling. Cell Signal. 2009;21(6):906-915.

13. Lissitzky JC, Parriaux D, Ristorcelli E, Verine A, Lombardo D, Verrando P. Cyclic AMP signaling as a mediator of vasculogenic mimicry in aggressive human melanoma cells in vitro. Cancer Res. 2009;69(3): 802-809. 
14. Meng J, Sun B, Zhao X, et al. Doxycycline as an inhibitor of the epithelial-to-mesenchymal transition and vasculogenic mimicry in hepatocellular carcinoma. Mol Cancer Ther. 2014;13(12): 3107-3122.

15. Qiao L, Liang N, Zhang J, et al. Advanced research on vasculogenic mimicry in cancer. J Cell Mol Med. 2015;19(2):315-326.

16. Liu Q, Qiao L, Liang N, et al. The relationship between vasculogenic mimicry and epithelial-mesenchymal transitions. J Cell Mol Med. 2016;20(9):1761-1769.

17. Hess AR, Seftor EA, Gardner LM, et al. Molecular regulation of tumor cell vasculogenic mimicry by tyrosine phosphorylation: role of epithelial cell kinase (Eck/EphA2). Cancer Res. 2001;61(8):3250-3255.

18. Yao L, Zhang D, Zhao X, et al. Dickkopf-1-promoted vasculogenic mimicry in non-small cell lung cancer is associated with EMT and development of a cancer stem-like cell phenotype. J Cell Mol Med. 2016;20(9):1673-1685.

19. Zhang S, Fu Z, Wei J, Guo J, Liu M, Du K. Peroxiredoxin 2 is involved in vasculogenic mimicry formation by targeting VEGFR2 activation in colorectal cancer. Med Oncol. 2015;32(1):414.

20. Liu X, Wang JH, Li S, et al. Histone deacetylase 3 expression correlates with vasculogenic mimicry through the phosphoinositide3kinase/ERK-MMP-laminin5 $\gamma 2$ signaling pathway. Cancer Sci. 2015;106(7):857-866.

21. Lo KW, Kan HM, Gagnon KA, Laurencin CT. One-day treatment of small molecule 8-bromo-cyclic AMP analogue induces cell-based VEGF production for in vitro angiogenesis and osteoblastic differentiation. J Tissue Eng Regen Med. 2016;10(10):867-875.

22. Sugimoto N, Miwa S, Tsuchiya H, et al. Targeted activation of PKA and Epac promotes glioblastoma regression in vitro. Mol Clin Oncol. 2013;1(2):281-285.
23. Ichikawa K, Kubota Y, Nakamura T, et al. MCRIP1, an ERK substrate, mediates ERK-induced gene silencing during epithelialmesenchymal transition by regulating the co-repressor CtBP. Mol Cell. 2015;58(1):35-46.

24. Keranen T, Hommo T, Moilanen E, Korhonen R. beta2-receptor agonists salbutamol and terbutaline attenuated cytokine production by suppressing ERK pathway through cAMP in macrophages. Cytokine. 2017;94:1-7.

25. Zhao N, Sun BC, Zhao XL, et al. Role of Bcl-2 and its associated miRNAs in vasculogenic mimicry of hepatocellular carcinoma. Int $J$ Clin Exp Pathol. 2015;8(12):15759-15768.

26. Vempati P, Popel AS, Mac Gabhann F. Extracellular regulation of VEGF: isoforms, proteolysis, and vascular patterning. Cytokine Growth Factor Rev. 2014;25(1):1-19.

27. Paulis YW, Soetekouw PM, VerheulHM, Tjan-Heijnen VC, Griffioen AW. Signalling pathways in vasculogenic mimicry. Biochim Biophys Acta. 2010;1806(1):18-28.

28. Huang B, Xiao E, Huang M. MEK/ERK pathway is positively involved in hypoxia-induced vasculogenic mimicry formation in hepatocellular carcinoma which is regulated negatively by protein kinase A. Med Oncol. 2015;32(1):408.

29. Fan YL, Zheng M, Tang YL, Liang XH. A new perspective of vasculogenic mimicry: EMT and cancer stem cells (review). Oncol Lett. 2013;6(5):1174-1180.

30. Kirschmann DA, Seftor EA, Hardy KM, Seftor RE, Hendrix MJ. Molecular pathways: vasculogenic mimicry in tumor cells: diagnostic and therapeutic implications. Clin Cancer Res. 2012;18(10):2726-2732.

31. Tang NN, Zhu H, Zhang HJ, et al. HIF-1alpha induces VE-cadherin expression and modulates vasculogenic mimicry in esophageal carcinoma cells. World J Gastroenterol. 2014;20(47):17894-17904.
OncoTargets and Therapy

\section{Publish your work in this journal}

OncoTargets and Therapy is an international, peer-reviewed, open access journal focusing on the pathological basis of all cancers, potential targets for therapy and treatment protocols employed to improve the management of cancer patients. The journal also focuses on the impact of management programs and new therapeutic agents and protocols on

\section{Dovepress}

patient perspectives such as quality of life, adherence and satisfaction The manuscript management system is completely online and includes a very quick and fair peer-review system, which is all easy to use. Visit http://www.dovepress.com/testimonials.php to read real quotes from published authors. 